\title{
VISIR Upgrade Overview and Status
}

\author{
Florian Kerber*a, Hans Ulrich Käufl ${ }^{\mathrm{a}}$, Pedro Baksai ${ }^{\mathrm{b}}$, Nicola Di Lieto ${ }^{\mathrm{a}}$, Danuta Dobrzycka ${ }^{\mathrm{a}}$, Philippe \\ Duhoux $^{\mathrm{a}}$, Gert Finger ${ }^{\mathrm{a}}$, Stefanie Heikamp ${ }^{\mathrm{d}}$, Derek Ives ${ }^{\mathrm{a}}$, Gerd Jakob ${ }^{\mathrm{a}}$, Lars Lundin ${ }^{\mathrm{a}}$, Dimitri Mawet ${ }^{\mathrm{b}}$, \\ Leander Mehrgan $^{\mathrm{a}}$, Yazan Momany ${ }^{\mathrm{b}}$, Vincent Moreau ${ }^{\mathrm{c}}$, Eric Pantin ${ }^{\mathrm{c}}$, Miguel Riquelme ${ }^{\mathrm{b}}$, Stefan \\ Sandrock $^{\mathrm{a}}$, Ralf Siebenmorgen ${ }^{\mathrm{a}}$, Alain Smette ${ }^{\mathrm{a}}$, Julian Taylor ${ }^{\mathrm{a}}$, Mario van den Ancker ${ }^{\mathrm{a}}$, Guillermo \\ Valdes $^{\mathrm{b}}$, Lars Venema ${ }^{\mathrm{e}}$, Ueli Weilenmann ${ }^{\mathrm{b}}$ \\ ${ }^{a}$ European Southern Observatory, Karl-Schwarzschild-Str. 2, 85748 Garching, Germany; \\ ${ }^{b}$ European Southern Observatory, Alonso de Córdova 3107, Vitacura, Santiago de Chile, Chile; \\ ${ }^{c}$ CEA Saclay, DSM/DAPNIA/Service d'Astrophysique, 91191 Gif-sur-Yvette, France \\ ${ }^{\mathrm{d}}$ Leiden Observatory, P.O. Box 9513, NL-2300 RA Leiden, The Netherlands \\ eASTRON, Oude Hoogeveensedijk 4, 7991 PD Dwingeloo, The Netherlands
}

\begin{abstract}
We present an overview of the VISIR upgrade project. VISIR is the mid-infrared imager and spectrograph at ESO's VLT. The project team is comprised of ESO staff and members of the original VISIR consortium: CEA Saclay and ASTRON. The project plan is based on input from the ESO user community with the goal of enhancing the scientific performance and efficiency of VISIR by a combination of measures: installation of improved hardware, optimization of instrument operations and software support. The cornerstone of the upgrade is the $1 \mathrm{k}$ by $1 \mathrm{k} \mathrm{Si}$ :As AQUARIUS detector array (Raytheon) which has been carefully characterized in ESO's IR detector test facility (modified TIMMI 2 instrument). A prism spectroscopic mode will cover the N-band in a single observation. New scientific capabilities for high resolution and high-contrast imaging will be offered by sub-aperture mask (SAM) and phase-mask coronagraphic (4QPM/AGPM) modes. In order to make optimal use of favourable atmospheric conditions a water vapour monitor has been deployed on Paranal, allowing for real-time decisions and the introduction of a user-defined constraint on water vapour. During the commissioning in 2012 it was found that the on-sky sensitivity of the AQUARIUS detector was significantly below expectations and that VISIR was not ready to go back to science operations. Extensive testing of the detector arrays in the laboratory and on-sky enabled us to diagnose the cause for the shortcoming of the detector as excess low frequency noise (ELFN). It is inherent to the design chosen for this detector and can't be remedied by changing the detector set-up. Since this is a form of correlated noise its impact can be limited by modulating the scene recorded by the detector. We have studied several mitigation options and found that faster chopping using the secondary mirror (M2) of the VLT offers the most promising way forward. Faster M2 chopping has been tested and is scheduled for implementation before the end of 2014 after which we plan to re-commission VISIR. In addition an upgrade of the IT infrastructure related to VISIR is planned in order to support burst-mode operations. The upgraded VISIR will be a powerful instrument providing close to background limited performance for diffraction-limited observations at an 8-m telescope. It will offer synergy with facilities such as ALMA, JWST, VLTI and SOFIA, while a wealth of targets is available from survey work (e.g. VISTA, WISE). In addition it will bring confirmation of the technical readiness and scientific value of several aspects of potential mid-IR instrumentation at Extremely Large Telescopes.
\end{abstract}

Keywords: VISIR, upgrade, ESO VLT, mid-IR, AQUARIUS, excess low frequency noise, chopping, water vapour

*fkerber@eso.org; phone +49 89 32006757; fax +49 89 3206530;

Ground-based and Airborne Instrumentation for Astronomy V, edited by

Suzanne K. Ramsay, lan S. McLean, Hideki Takami, Proc. of SPIE Vol. 9147,

91470C · ( 2014 SPIE · CCC code: 0277-786X/14/\$18 · doi: 10.1117/12.2055172

Proc. of SPIE Vol. 9147 91470C-1 


\section{INTRODUCTION}

VISIR is the mid-IR imager and spectrograph at ESO's Very Large Telescope (VLT) ${ }^{1}$. It was built by a French-Dutch consortium (Service d'Astrophysique CEA and ASTRON - PI: P.O. Lagage, Co-PI: J.W. Pel) and has been operational since the end of 2004. It is located at the Cassegrain focus of unit telescope (UT) 3 (Melipal) at VLT on Cerro Paranal $(2635 \mathrm{~m})$ in Northern Chile. The instrument provides diffraction-limited imaging at high sensitivity in the two midinfrared (mid-IR) atmospheric windows: the N-band between 8 to $13 \mu \mathrm{m}$ and the Q-band between 16.5 and $24.5 \mu \mathrm{m}$. In addition, it features a long-slit spectrometer with a range of spectral resolutions between 150 and 30,000.

The upgrade of VISIR has been described at previous SPIE conferences ${ }^{2,3}$. For convenience some of the relevant information is repeated and then an update on the recent developments will be given.

For technical details of the instrument and its use we refer to the VISIR homepage at ESO: http://www.eso.org/sci/facilities/paranal/instruments/visir/ and to the description of the instrument by the consortium ${ }^{1}$. Updates on the upgrade status are also available on a dedicated web page (http://www.eso.org/sci/facilities/paranal/instruments/visir/upgradeproject.html). ESO issues a call for proposals twice a year; preparation of proposals asking for observing time with VISIR is supported by an exposure time calculator: http://www.eso.org/observing/etc/.

The goals of the upgrade are summarized in the following list:

- Improved performance of existing modes: achieve background limited performance for imaging and lowresolution spectroscopy

- Optimise use of observing time: more efficient N-band spectroscopy, knowledge of atmospheric conditions, in particular precipitable water vapour (PWV)

- Provide new science capabilities: new modes for high contrast and high resolution imaging

- Expand support of data analysis software in line with enhanced capabilities and ESO policies

- Conduct upgrade in close collaboration with Paranal and partners from the original consortium

\section{AQUARIUS DETECTOR ARRAY}

ESO funded the development of a new Si:As Impurity Band Conduction (IBC) array, named AQUARIUS, at Raytheon Vision Systems, Santa Barbara, USA. Five science devices, an engineering grade and multiplexers have been delivered to ESO, as part of this contract. AQUARIUS has $1024 \mathrm{x} 1024$ pixels, each $30 \mu \mathrm{m}^{2}$ in size and is therefore approximately five to six times larger in area than the previous generation of high background mid-IR detectors.

The architecture of the detector is such that it is split into two areas each of 512 rows and 1024 columns, at the top and bottom of the device. Each area has 32 outputs, such that, each output is configured to read out $32 \times 512$ pixels, all 64 outputs from the two areas being read in parallel. This readout scheme also allows for 16 outputs rather than 64 to simplify system design for low background applications. With this multiplexer configuration it is possible to read the detector out at $150 \mathrm{~Hz}$ frame rates, each output operational at $3 \mathrm{MHz}$ pixel rates. A windowed readout is possible, where a user selectable number of rows can be read, from the center outwards, with the remaining rows reset automatically. For example, a 1024 x 150 sized window, centred in the middle of the device, can be readout at $1 \mathrm{kHz}$ frame rates. There is no advantage to windowing in the column direction since all outputs run in parallel.

A more detailed description of the AQUARIUS array and its performance is given by Ives et al. ${ }^{4}$ at this SPIE conference. The following parameters characterize the performance of the AQUARIUS based on laboratory testing: 
Table 1 - AQUARIUS detector specification and performance summary.

\begin{tabular}{|l|l|l|}
\hline \multicolumn{1}{|c|}{ Specification } & \multicolumn{1}{c|}{ Measured } & \multicolumn{1}{c|}{ Comment } \\
\hline Array size & $1024 \mathrm{x} 1024$ pixels & Pixel size is $30 \mu \mathrm{m}$ \\
\hline Operating temperature & $7-9 \mathrm{~K}$ & 9K typically used \\
\hline Frame Rate & $>100 \mathrm{~Hz}$ & $>150 \mathrm{~Hz}$ possible and also windowing \\
\hline Spectral Response & $3-28 \mu \mathrm{m}$ & Our AR coating optimised at $6 \mu \mathrm{m}$ \\
\hline Quantum Efficiency & $>40 \%$ & Measured indirectly, see note later \\
\hline Input referred noise & $\sim 200 \mathrm{e}-\mathrm{rms}$ & For single read, $\mathrm{kTC}$ noise not dominant \\
\hline Power dissipation & $\sim 250 \mathrm{~mW}$ & For 64 outputs \\
\hline Full well & $\sim 0.8 \mathrm{Me}$ & High gain mode \\
\hline Dark current & $1 \mathrm{e} / \mathrm{pixel} / \mathrm{second}$ & Measured at $7 \mathrm{~K}$ \\
\hline Non-linearity & $<5 \%$ & Better than $0.1 \%$ over $2 / 3$ full well (corrected) \\
\hline Crosstalk & $\sim 6 \%$ to adjacent pixels & Measured using stochastic method \\
\hline
\end{tabular}

\section{VISIR INTERVENTION AND INSTALLATION OF NEW HARDWARE}

Following the last official close-out night of "old VISIR" on $7^{\text {th }}$ May 2012, the complete instrument was dismounted from VLT UT3 and brought to the integration hall of La Silla Paranal Observatory. In May and June the optical instrument was disassembled and prepared for the integration of new hardware. Imager and spectrometer detectors were equipped with the AQUARIUS array integrated in dedicated detector mounts. Both detectors could be adjusted to optimum focus. Preliminary overall detector system performance testing in the range of $5.5 \mathrm{~K}-10 \mathrm{~K}$ revealed optimized detector operation around $8.5 \mathrm{~K}$.

A second major upgrade task was the implementation of a prism optics in the VISIR spectrometer. One of four gratings on the carousel wheel was replaced by a ZnSe prism with dedicated cryogenic mount. VISIR's spectrograph arm uses two collimators, one for high-resolution Echelle spectroscopy, the other for low and medium resolution work. We have replaced the low resolution gratings with one singe $\mathrm{ZnSe}$ prism, which will allow for long-slit spectroscopy in the Nband with a spectral resolution $\lambda / \Delta \sim 500$ at $\lambda \sim 8 \mu \mathrm{m}$ and $\sim 800$ at $\lambda \sim 13 \mu \mathrm{m}$ in one exposure, hence providing a gain of four in observing efficiency for the full N-band spectrum. This mode will have both uncompromised through-put - no order sorting filter - and very good image quality, as a prism will perform much better than a grating. The upgrade of the lowresolution mode will give a boost to spectrometric differential observing techniques.

\subsection{New Scientific modes}

\section{The VISIR coronagraphic mode}

This mode has been primarily designed to observe point sources (companion stars, brown dwarfs) at narrow angular separations from the primary $(0.3-1 \mathrm{arcsec})$ and resolve the central regions of dusty disks (proto-planetary and debrisdisk) at the same angular separations. Three devices have been installed in VISIR, two 4-quadrants phase masks (4QPM) developed in the framework of the MIRI imager ${ }^{5}$, and one achromatic AGPM (Annular Groove Phase Mask, a vortex coronagraph $^{6}$. The germanium-based $4 \mathrm{QPMs}$ are chromatic and are designed to work at a relatively narrow wavelength (10.5 and the $11.3 \mu \mathrm{m}, \delta \lambda=0.4$ and $0.6 \mu \mathrm{m}$, respectively) corresponding to spectral features of astrophysical interest (e.g. the $11.3 \mu \mathrm{m}$ PAH emission band and adjacent continuum). The diamond-plate AGPM provided by the University of Liege (IAGL) is optimized to work on a broader range $(12.25 \mu \mathrm{m}$ central wavelength, $\delta \lambda=1.3 \mu \mathrm{m})$ and covers the [NeII] emission line at $12.8 \mu \mathrm{m}$. The coronagraphic masks have been implemented at the VISIR focal plane after the entrance window. Dedicated Lyot stops have been designed and placed in front of the corresponding filters, in the pupil plane. 


\section{The sub-aperture mode (SAM)}

This mode allows to bridge the gap between the mono-pupil imaging mode and the interferometric instruments (e.g VLT/MIDI). A pupil plane mask combined with a filter is implemented in a free slot of the filter wheel. This nonredundant mask ${ }^{7}$ contains 7 holes building a network of 21 baselines. The integrated transmission is about $25 \%$. This mode is particularly well suited to overcome the moderate phase errors due to the infrared seeing. It is optimized to detect faint extensions or companions around bright objects in the $0.5-2 \lambda / \mathrm{D}$ distance range (0.1-0.4 arcsec), see e.g. Monnier ${ }^{8}$. Two wavelengths/filters have been implemented: 10.5 and $11.3 \mu \mathrm{m}, \delta \lambda=0.4$ and $0.6 \mu \mathrm{m}$ respectively. The masks were cut in aluminum 508T6 thin plate and covered with an absorbing layer obtained after an anodization process.

\section{COMMISSIONING IN 2012}

During commissioning all hardware exchanges were successfully implemented. The optical quality is excellent and all modifications including the new prism based low-resolution spectroscopy work well. Offline and on-sky tests of the new modes (coronagraphs and SAM-imaging) were very encouraging.

Early in commissioning observations of standard stars showed that the on-sky sensitivity of VISIR with the new AQUARIUS detector fell short of the requirements and was in fact about a factor 2-3 smaller than the one of the old instrument with the Boeing DRS detector (Figure 1). This statement holds for any optical configuration and observing mode. This came as a complete surprise since no indication of a problem had been found during laboratory testing.

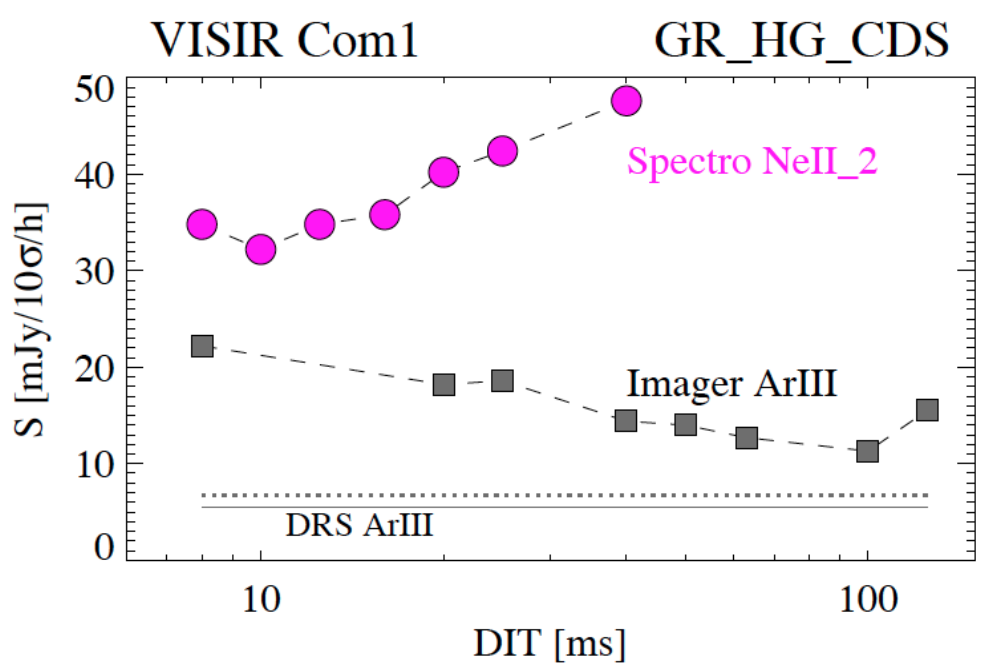

Figure 1. Sensitivity of the imager detector in ArIII filter (grey) and of the spectrometer (magenta) in imaging mode using the NeII_2 filter. For comparison the sensitivity of the old VISIR using the DRS detectors are shown for the imager, median of all data obtained with good weather shown as dotted line and the top $25 \%$ sensitivity of the DRS as solid line.

We had to conclude that VISIR was not ready for returning to science operations and a dedicated plan was set up to identify the root cause of the lack of sensitivity. 


\section{TESTS AND DIAGNOSIS OF PROBLEM}

\subsection{Detector Quantum Efficiency (QE)}

Absolute detector response values are difficult to obtain. For the AQUARIUS detector there were no reliable quantum efficiency values available. When the commissioning of VISIR showed a lack of sensitivity, we decided to invest dedicated effort in evaluating the detector quantum efficiency. We used a calibrated blackbody (BB) radiation source which provides a known photon flux to the AQUARIUS detector mounted in the focal plane of the mid-IR test facility. With this set-up the product of AQUARIUS QE and throughput (TP) of the test facility gave a $20 \%$ response. For the absolute determination of the test facility's optical throughput we used a calibrated pyroelectric detector in place of the AQUARIUS detector. The test facility optical throughput TP was derived from the quotient of the pyroelectric signals of the calibration measurement and reference measurement: TP $=0.5 \pm 1.5 \%$. This includes the transmission and reflectance all four optical components of the cryo-vacuum instrument like KRS5-entrance window, two reflecting mirrors and one Ge-lens. With the known throughput value we calculated the quantum efficiency of the AQUARIUS detector to be $40 \pm 1.5 \%$ a value which is within expectations.

\subsection{Excess low frequency noise (ELFN)}

As a result of extensive testing we were able to identify the root cause for the limited sensitivity: excess low frequency noise. This phenomenon was described 30 years ago by Stapelbroek et al. ${ }^{9}$. The ELFN is a form of correlated noise caused by fluctuations in the space charge induced by ionization/recombination in the blocking layer. It manifests as a memory of photons in subsequent frames. This correlation can be broken by modulating the scene (sources and background) seen by the detector at sufficiently high speed (Fig. 2). Since the AQUARIUS array was originally developed for space applications with extremely low background (JWST/MIRI) it has a thick blocking layer and hence this design results in significant ELFN in high background conditions which has a massive impact on sensitivity in the ground-based (high background) case for VISIR (Fig. 2). This was not properly accounted for in the design of the Si:As detector material hybridized on the AQUARIUS multiplexer for the ESO contract. For future ground-based devices a better design will be warranted. The ELFN can't be mitigated at the detector level. A full analysis is given in Ives et al. ${ }^{4}$.

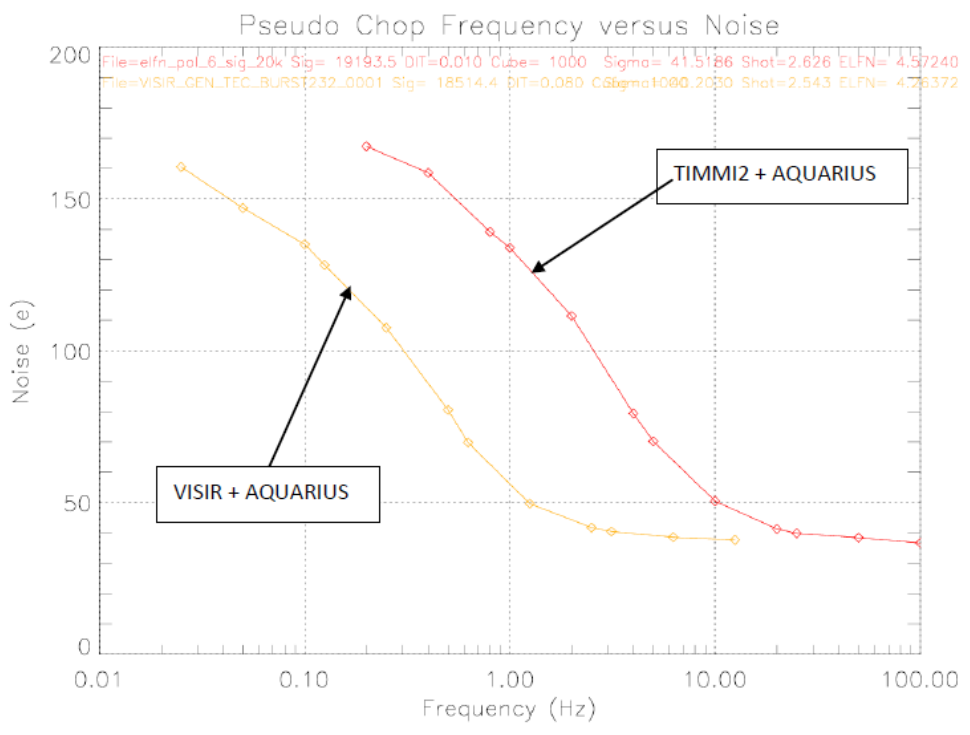

Figure 2. Noise versus frequency of the SIV filer measured in the lab with IR test facility using Aquarius device FLT112 (red) and on-sky with VISIR imager detector FLT109 (orange). 


\section{SUMMARY OF TECHNICAL TESTS IN AUGUST 2013}

In August 2013 we used 4 half nights of technical time to validate the performance of the AQUARIUS detector array and explore feasibility of the potential mitigation options in order to recover VISIR's on-sky sensitivity to the extent possible. We tried faster chopping with the M2 of the VLT as well as drift scanning (sections 7.3 \& 7.2). The average optical seeing during the observations was 1". This results in a degradation of PSF sharpness when compared to the theoretical diffraction limit. In burst mode data one can correct for the PSF sharpness loss by applying a shift-and-add correction on the individual exposures. The results are shown in Figure 3. B10.7 is a broad-band filter centered at 10.7 $\mu \mathrm{m}$. It represents a worst case in terms of ELFN impact. A frequency of a $2-3 \mathrm{~Hz}$ will be required to reach $75 \%$ of the background-limited performance for broad-band filters. For narrow-band filters and spectroscopy which are less affected by ELFN lower frequencies will already be suitable. Both faster M2 chopping and drift scanning are able to control the impact of ELFN and recover on-sky sensitivity. Other aspects of detector read-out, cosmetics and artifacts were also tested and good performance in these aspects has been demonstrated.

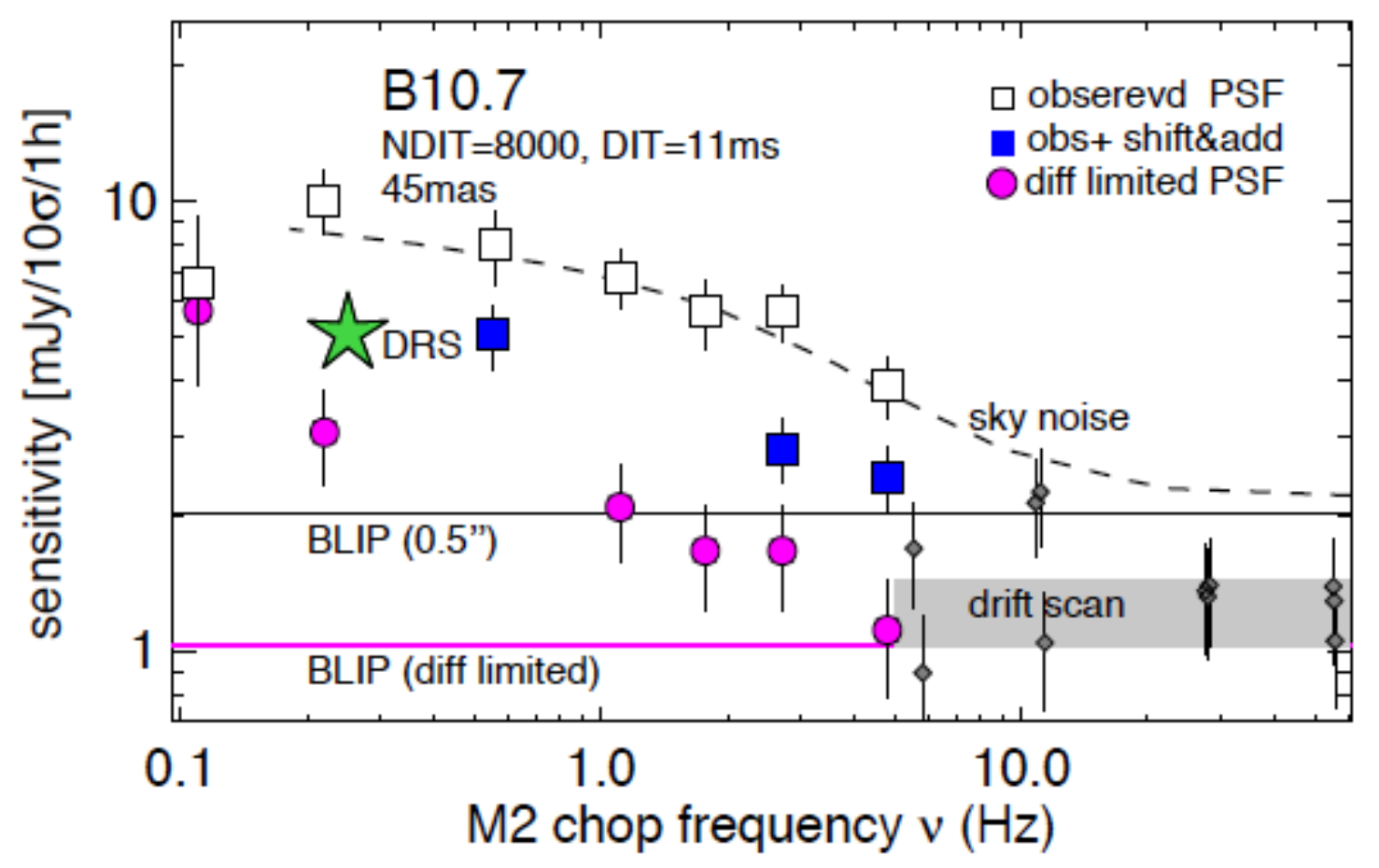

Figure 3. VISIR sensitivities as a function of chopping frequency. Observations are obtained in burst mode and filter B10.7 on the standard star HD198048. Sensitivities are deduced from 3 types of data processing: i) standard imaging mode observation. These are affected by seeing and image quality problems (open squares), ii) applying a shift-and-add correction of the jitter image (blue squares), and iii) estimating the noise from the background. This sensitivity estimate approximates best diffraction limited performance (magenta circles). For comparison the median sensitivity of filter B10.7 with the DRS detector is marked by a green star. Sensitivities derived from drift scans located at equivalent chopping frequencies (derived from the scan speed) are shown as grey dots. The grey shaded area gives their 1-sigma range. Error bars reflect $1 \sigma$ uncertainties and are derived from the variance of the noise. The sky noise extracted from other measurements is scaled to match the $1 \mathrm{~Hz}$ data point. This dashed line gives the expected sensitivity gain with higher chopping frequency. Theoretical sensitivity limits (BLIP) are shown for 0.5 " seeing (solid line) and for diffraction limited observations (magenta line). 


\section{POTENTIAL MITIGATION MEASURES FOR AQUARIUS ARRAY}

\subsection{External chopper}

We have studied two concepts for an external chopper: post-focal chopping in the pupil plane and using a Dicke switch. The first one will be able to provide chopping frequencies up to $20 \mathrm{~Hz}$ whereas the second will be limited to less than 5 Hz. Both options will involve significant cost and effort. Since mass and volume constraints at the Cassegrain focus are tight both would be difficult to implement at the VLT. Given the fact that chopping up to a few $\mathrm{Hz}$ is feasible using the VLT's M2 and that such frequencies should be sufficient to control the AQUARIUS noise an external chopper is not part of the VISR upgrade project. It is worth noting that a cryogenic chopper is part of the METIS instrument design for the E-ELT. Details of the development and characterization of such a cryogenic device was presented at this conference ${ }^{10}$.

\subsection{Drift-scanning}

In this method the object is consciously moved across the FoV during the observation, hence the scene observed by the detector gets modulated by this motion. Details of the approach have been presented at this conference by Heikamp et al. ${ }^{11}$, also with a view to application for future E-ELT instrumentation. The effectiveness of this approach for the AQUARIUS array has been demonstrated during on-sky testing in Aug 2013 (see Fig. 3). The implementation at the VLT though would require a major modification of the telescope control system and for this reason we do not plan to pursue this option for the VISIR upgrade.

\subsection{Faster chopping with VLT}

From the laboratory tests (Fig. 2) and on-sky testing (Fig. 3) it is evident that faster chopping will limit the impact of the ELFN (section 6). A frequency of 2-3 Hz will be adequate to approach background-limited performance for many filters in good seeing conditions. Hence, this has been adopted as primary option for VISIR upgrade to mitigate the effect of ELFN and recover on-sky sensitivity. The technical implementation of faster chopping has been tested during technical time at the VLT and will be described in more detail below.

\section{FASTER M2 CHOPPING AS MITIGATION MEASURE FOR AQUARIUS ARRAY}

During technical time on the VLT (March 2014) we conducted tests of the M2 field stablization control loop and the read-out scheme of the new technical CCD (NTCCD).

\subsection{Current read-out scheme}

The current read-out scheme of the NTCCD is shown in Figure 4.

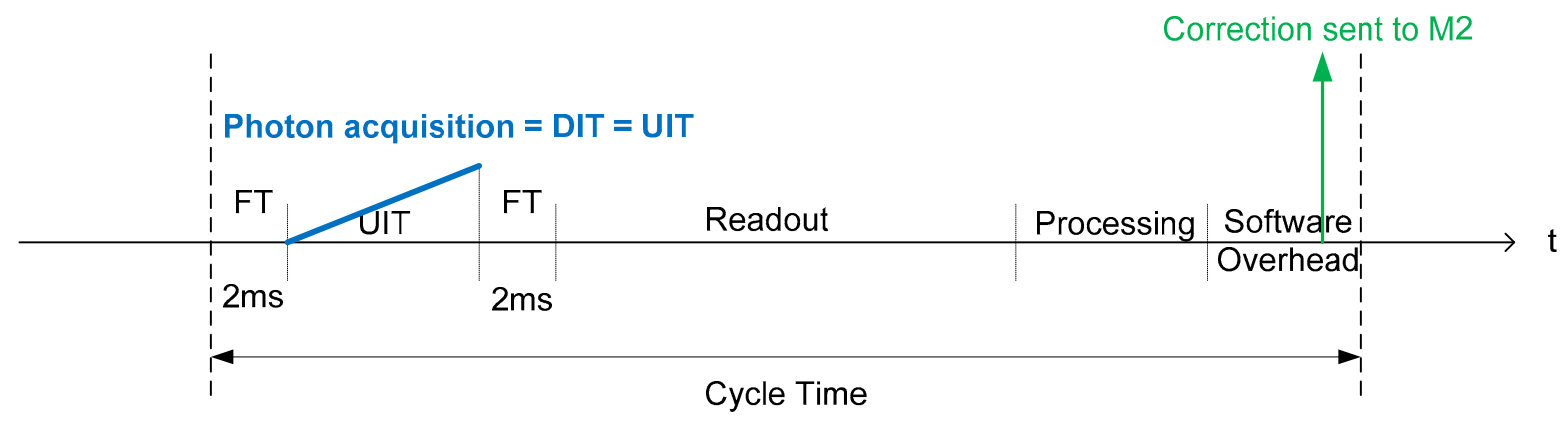

Figure 4. Classical timing diagram of a field stabilization period 
The current standard DIT is of order of 20 ms providing a duty cycle with an efficiency of 50-65\%. When shorter DITs are to be used the efficiency drops significantly because of the sequential nature of the readout process.

\subsection{New read-out scheme}

In the new scheme we take advantage of the frame transfer capability of the CCD type CCD57-10 by e2VTM. Hence the read-out becomes a continuous process resulting in a significantly increased efficiency of above $87 \%$ even at fast rates. The minimum exposure time is imposed by the software and processing overhead.

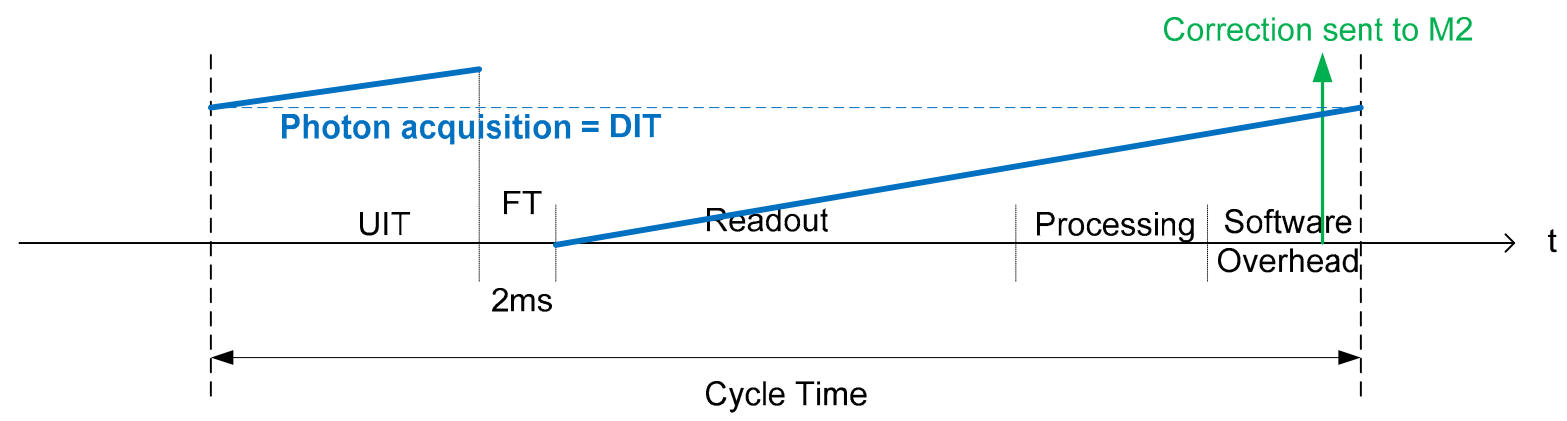

Figure 5. Pipelined timing diagram of a field stabilization period

The performance of FS using the new scheme has been tested and very good performance has been confirmed. Chopping up to $4 \mathrm{~Hz}$ has been demonstrated and no significant degradation of the FS performance can be reported when comparing the chopping at 1 and $2 \mathrm{~Hz}$ on a GS of magnitude 12, 13 and $14^{\text {th }}$ magnitude. However the data collected during the test show a strong degradation of the FS quality (RMS increase of a factor 2.5) when the chopping frequency increases from 3 to $4 \mathrm{~Hz}$. The percentage of corrections per phase is then about 40\% (5 to 6 corrections) which seems to be the lower limit.

\subsection{Modified field stabilization control loop}

A simulation model of the FS loop was built. A critical part of the model is the dynamic response of M2, which was measured on UT1 by injecting test signals and logging data. The closed loop controller was found to be tuned so aggressively that it becomes unstable at high TCCD frame rates. An alternative, less aggressive tuning was calculated in simulation and validated experimentally up to $62 \mathrm{~Hz}$ frame rate, which allowed chopping at up to $4 \mathrm{~Hz}$ on sky.

It is important to note that excellent agreement between the model and the actual performance of the FS loop has been demonstrated both on the currently implemented loop and the improved loop with new parameters.

\section{Analysis of FS loop}

The measurements agree well with the predictions of the Simulink model and confirm that the loop is quite aggressively tuned and increasingly so for higher frame rates.

As the frame rate is increased, two "bumps" grow in the rejection transfer function, one centred around $8 \mathrm{~Hz}$ and a second centred around $30 \mathrm{~Hz}$ (the bump frequencies also shift depending on the frame rate).

The model predicts the onset of instability between $50-55 \mathrm{~Hz}$ frame rate. Experimentally, frame rates of 27, 38, 48, 56 and $59 \mathrm{~Hz}$ were attempted (corresponding to 20, 10, 5, 2 and $1 \mathrm{~ms}$ DIT). 
With the original gains $(\mathrm{Kp}=1.2, \mathrm{Ki}=2.5, \mathrm{Kii}=0)$ the amplification of disturbances is at the limit of acceptability $(+10 \mathrm{~dB})$ at $48 \mathrm{~Hz}$ frame rate (5 ms DIT, Fig. 6, red line); the loop is unstable at 56 and $59 \mathrm{~Hz}$, in accordance with the model.

New less aggressive gains $(\mathrm{Kp}=0.7, \mathrm{Ki}=1.5, \mathrm{Kii}=0)$ were synthesized in simulation and verified to work well on sky at all frame rates. As an example, the maximum amplification in the response at $48 \mathrm{~Hz}$ frame rate $(5 \mathrm{~ms}$ DIT, Fig. 6, black line) is reduced by approximately $5 \mathrm{~dB}$.

\subsection{Findings}

- The model based approach for understanding and tuning the FS control loop was successful and the high predictive power of the model was demonstrated.

- The existing field stabilization controller gains were found to be too aggressive at high frame rates which results in high disturbance amplification and even instability.

- New less aggressive gains were tested on sky and allowed stable operation at all frame rates up to $62 \mathrm{~Hz}$ and chopping frequencies up to $4 \mathrm{~Hz}$, whereby a significant reduction of the FS performance is found above $3 \mathrm{~Hz}$.

- An alternative, parallel readout mode for the new TCCD taking advantage of the frame-transfer capability has successfully been tested. It is fully compatible with the modified FS control loop and enables operation on fainter guide stars at high frame rates.

The new gains also allow stable operation with the alternate parallel readout mode, which consists in letting the CCD integrate after the frame transfer and during the readout.

The parallel readout mode allows maximizing the frame rate $(62 \mathrm{~Hz})$ with a DIT of $14 \mathrm{~ms}$, compared to $59 \mathrm{~Hz}$ and DIT 1 ms of the conventional mode. Therefore it is particularly well suited for guiding on faint stars at high frame rates.

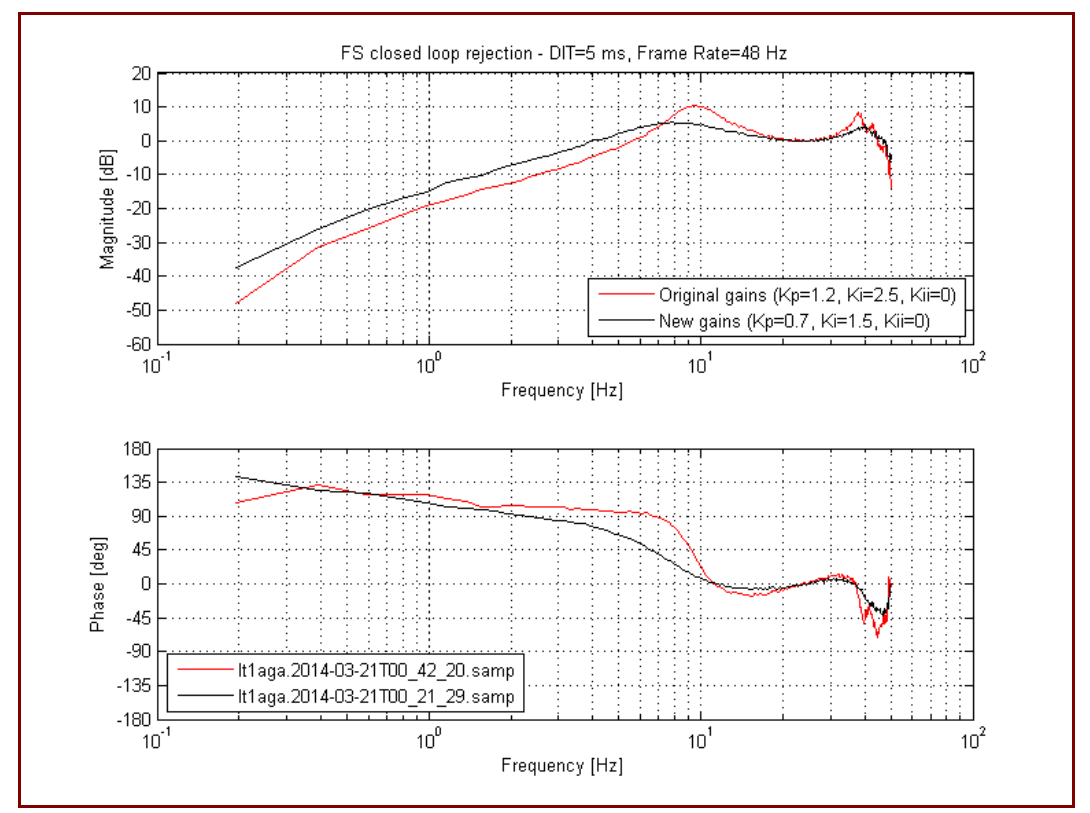

Figure 6. Field Stabilization rejection - Comparison new (black) vs. original (red) gains at $48 \mathrm{~Hz}$ frame rate.

Note the higher slope of the phase around $9 \mathrm{~Hz}$ with the original gains, which is an indicator of resonance. 


\section{SUPPORT FOR VISIR OPERATIONS}

\subsection{Software}

The VISIR upgrade poses several challenges to the existing data reduction software (pipeline). The new detector is 16 times larger which increases the memory usage, processing time and disk space requirements significantly. Also the use of burst mode increases the amount of data the pipeline needs to handle by several magnitudes. To cope with this data rate the pipeline makes use of multiple CPU cores and vector extensions available on most modern machines and uses highly optimized third party libraries like fftw $3^{12}$ for performance critical tasks. The software has been developed and tested successfully.

\subsection{Infrastructure}

As part of the VISIR upgrade, the old 256x256 pixel DRS detectors of VISIR have been replaced with 1024x1024 pixel Raytheon AQUARIUS detectors. The increase in format will imply an increase by a factor of 16 of the data volume produced by VISIR compared to the old situation. For low-, high- and medium-resolution spectroscopy and for regular imaging observations, this increase in data volume is not expected to pose any significant challenges for the VLT data flow infrastructure.

However, if run in the full burst mode (in which one frame is saved per Detector Integration Time (DIT)), VISIR will be capable of producing data at a rate of 262 Mbyte/second, or about 5-6 Tbytes per night. This rate exceeds the current limits set by the transfer speeds, storage capacity, and pipeline processing speeds of the VLT data flow system. Therefore we plan to modify the implementation of the burst mode, such that no longer each DIT is saved, but one frame is saved for each DITxNDIT (with NDIT a user-defined parameter). DITxNDIT will be set to values equal or larger than $30 \mathrm{~ms}$ to stay within the limitations of the VLT data flow system. This will allow all data to be transferred in a shorter time than the total integration time (avoiding data-transfer bottle-necks), and reduce the amount of data which needs to be stored to less than 1.5 Tbyte/night. Windowing of the data may be a good alternative for some programmes to run the burst mode at the full frame rate, but as this will significantly reduce the available field-of-view, this not a general solution for all programmes. Hence the implementation of this option has a lower priority than the averaging of DITs.

An upgrade of the current pipeline and offline machines in Paranal to Blade M620 or M820 machines with a 10 Gbits/second ethernet interface and a high-performance hard-disk with a capacity larger than 5 TBytes is also planned to be able to store VISIR burst-mode data, and to run the VISIR data-reduction pipeline in a reasonable amount of time (necessary to do quality control on the data, and hence offer the burst mode for service-mode observations). In addition, an upgrade of the ethernet interfaces of all the existing workstations involved in the transfer of VISIR data to 10 Gbits/second are planned in order to take full advantage of the existing network infrastructure.

\section{SUPPORT OF SCIENCE OPERATIONS: PWV}

A water vapour monitor has been permanently deployed at ESO's Paranal observatory as part of the VISIR upgrade project. The instrument selected is a Low Humidity And Temperature PROfiling microwave radiometer (LHATPRO), manufactured by Radiometer Physics GmbH (RPG). The unit measures several channels across the strong water vapour emission line at $183 \mathrm{GHz}$, necessary for resolving the low levels of precipitable water vapour (PWV) that are prevalent on Paranal (median $\sim 2.5 \mathrm{~mm}$ ). The unit comprises the above humidity profiler (183-191 GHz), a temperature profiler $(51-58 \mathrm{GHz})$, and an infrared radiometer $(\sim 10 \mu \mathrm{m})$ for cloud detection. Details of the instrument and its operations have been presented at a previous SPIE ${ }^{13}$.

Water vapour is one of the main sources of opacity in the Earth's atmosphere in the thermal infrared, the operating range of VISIR. The new PWV radiometer is a major upgrade over existing capabilities since it does not use on precious time on the UTs and also it provides much better time coverage and higher accuracy compared to the spectroscopic methods used previously ${ }^{14}$. Moreover, the PWV content of the atmosphere above Paranal is strongly variable, both on short 
timescales, and with pronounced seasonal variations. However, not all infrared observations are equally affected by the PWV conditions: whereas imaging and spectroscopy in the Q-band atmospheric window from 17-21 $\mu \mathrm{m}$ will strongly benefit from being done under conditions of relatively low water vapour, imaging in the N-band window $(9-12 \mu \mathrm{m})$ would be less sensitive to PWV contents. The introduction of PWV as a user defined constraint - similar to seeing or sky transparency in the optical - provides the opportunity to optimize the scientific return of infrared instruments like VISIR by matching the PWV needs of each observation carried out in service mode to the actual conditions measured in realtime by the PWV monitor. Furthermore, the addition of a real-time PWV monitoring capability to Paranal infrastructure will enable qualitatively new types of science projects that require conditions of particularly low $\mathrm{PWV}^{15}$ to be successfully executed.

\section{SUMMARY AND OUTLOOK}

The VISIR upgrade project has recovered from a major set-back that was discovered during commissioning in 2012. The on-sky sensitivity of the instrument with the new AQUARIUS detector was found to be 2 to 3 times worse than with the old detector. During extensive testing we have been able to identify the root cause of the problem: excess low frequency noise. This phenomenon first described 30 years ago has not been properly accounted for in the design of the AQUARIUS for the ground-based - high background - application in VISIR. The problem can't be resolved at the detector level and several mitigation options have been explored. We have demonstrated on-sky that faster chopping or drift scanning both will limit the impact of the ELFN. For the VISIR upgrade project we plan to implement chopping with the M2 of the VLT with frequencies of up to $4 \mathrm{~Hz}$. Feasibility and performance of improved field stabilization and chopping has been demonstrated on-sky taking advantage of an optimized field stabilization loop and a new read-out scheme of the technical CCD for guiding. We plan to fully commission this scheme in Nov 2014 and commission VISIR after that. The new VISIR will then provide to the ESO user community better sensitivity than the old VISIR. Operations will support burst mode offering the opportunity for frame selection bringing background limited performance within reach for most filters. Sub-aperture mask and coronagraphy will provide new scientific capabilities. In low resolution spectroscopy full wavelength coverage of the N-band is provided while for high resolution spectroscopy the free spectral range is increased by a factor 3. Finally, the users can take advantage of favourable atmospheric conditions and can specify constraints on the precipitable water vapour enabling very demanding observations. When all of these steps are combined and put into action VISIR will have been truly upgraded. The new VISIR will also be an important milestone for demonstrating technology and observing modes for future instrumentation at extremely large telescopes.

\section{REFERENCES}

[1] Lagage, P. O., Pel, J. W., Authier, M., et al. "Successful commissioning of VISIR: The mid-infrared VLT Instrument" The Messenger 117, 12-16 (2004).

[2] Kerber, F. Kaeufl, H.U., van den Ancker, M., et al. "Upgrade of VISIR the mid-infrared instrument at the VLT" Proc SPIE 7735, 7735R-7735R-12 (2010).

[3] Kerber, F. Kaeufl, H.U., Baksai, P., et al. "VISIR upgrade overview and status" Proc SPIE 8446, 84460E184460E12 (2012).

[4] Ives, D., Finger, G., Jakob, G., Beckmann, U. "AQUARIUS, the next generation mid-IR detector for ground based astronomy, an update" Proc SPIE 9154, 48 (2014).

[5] Rouan, D., Boccaletti, A., Baudoz, P., Cavarroc, C., Baudrand, J., Reess, J.M., “The Coronagraphic Mode of MIRI/JWST", proceedings of the conference In the Spirit of Bernard Lyot: The Direct Detection of Planets and Circumstellar Disks in the 21st Century. June 04 - 08, 2007. University of California, Berkeley, CA, USA. Edited by Paul Kalas. (2007).

[6] Mawet, D., Riaud, P., Absil, O., Surdej, J., “Annular Groove Phase Mask Coronagraph” ApJ 633, 1191-1200 (2005).

[7] Tuthill, P. G., Monnier, J. D., Danchi, W. C., "Aperture masking interferometry on the Keck I Telescope: new results from the diffraction limit” Proc. SPIE Vol. 4006, 491-498 (2000). 
[8] Monnier, J. D., Tuthill, P. G., Ireland, M., et al., "Mid-Infrared Size Survey of Young Stellar Objects: Description of Keck Segment-Tilting Experiment and Basic Results" ApJ 700, 491-505 (2009).

[9] Stapelbroek, M. G., Petroff, M. D., Speer, J. J., Bharat, R., "Origin of excess low frequency noise at intermediate infrared backgrounds in BIB detectors", Proc. IRIS Detector, No.2 (1984).

[10] Paalvast, S.L., Jassen, H., Teuwen, M., et al. "Development and characterization of a 2D precision cryogenic chopper for METIS" Proc SPIE 9151, 12 (2014).

[11] Heikamp, S., Brandl, B., Keller, C. U., et al. "Drift scanning technique for mid infrared background subtraction" Proc SPIE 9147, 372 (2014).

[12] http://www.fftw.org/

[13] Kerber, F., Rose, T., Chacon, A., et al. "A water vapour monitor at Paranal Observatory," in SPIE Conference Series 8446, 84463N (2012).

[14] Querel, R.R., Naylor, D.A., Kerber, F. "Spectroscopic Determination of Atmospheric Water Vapor" PASP 123, 222-229 (2011).

[15] Kerber, F., Querel, R. R., Rondanelli, R. et al. "An episode of extremely low precipitable water vapour over Paranal observatory” MNRAS 439, 247-255 (2014). 